\title{
ACTITUDES DE LOS GERENTES DE LOS ALOJAMIENTOS RURALES HACIA EL DESARROLLO DE UN TURISMO SOSTENIBLE
}

\author{
María Moral Moral* \\ María Teresa Fernández Alles* \\ Universidad de Cádiz \\ Manuel Jesús Sánchez Franco** \\ Universidad de Sevilla
}

\section{RESUMEN}

La finalidad de esta investigación es identificar las actitudes e intenciones del gerente de alojamientos rurales hacia la implementación de acciones socialmente responsables, y en consecuencia hacia un turismo sostenible. El carácter novedoso radica en la conexión entre la Teoría del Comportamiento Planeado (TCP) y la Teoría del Desarrollo Sostenible. La metodología empleada es la técnica de Partial Least Square, (PLS). Los resultados evidencian que la implementación de medidas sostenibles está determinada principalmente por las presiones del entorno del gerente.

Palabras clave: turismo sostenible; alojamiento rural; actitudes; Teoría del Comportamiento Planeado (TCP).

\section{Attitudes of rural accommodation managers towards the development of sustainable tourism}

\section{ABSTRACT}

The purpose of our research lies in identifying rural-hospitality managers' attitudes and intentions towards the implementation of social-responsability actions, and consequently

Recibido: 26 de diciembre de 2016

Devuelto para su revisión: 13 de junio de 2017

Aceptado: 4 de septiembre de 2017

Departamento de Marketing y Comunicación. Facultad de Ciencias Económicas y Empresariales. Universidad de Cádiz. C/ Enrique Villegas Vélez, 2. 11002 CÁDIZ (España). E-mail: maria.moral@uca.es ; teresa.alles@uca.es

** Departamento de Administración de Empresas y Marketing. Facultad de Ciencias Económicas y Empresariales. Universidad de Sevilla. Avda. Ramón y Cajal, 1. 41018 SEVILLA (España) E-mail: majesus@us.es 
toward sustainable tourism. The key research focus is on the association between the Theory of Planned Behavior and the Theory of Sustainable Development. Partial Least Square (PLS) is proposed to assess the relationships between the constructs together with the predictive power of the research model. Overall, our results conclude that the implementation of 'sustainable actions' is mainly determined by the pressures of the manager's environment.

Keywords: sustainable tourism; rural housing; attitudes; Theory of Planned Behavior (TCP).

\section{INTRODUCCIÓN}

Según la Organización Mundial de Turismo (en adelante, O.M.T) en el año 2015 un total de 1.184 millones de turistas viajaron por diferentes regiones del mundo; lo que representa un incremento del 4,4\% respecto al año anterior (O.M.T., 2016a). En este contexto, y atendiendo a las influencias del turismo en la sociedad, las instituciones públicas se han propuesto enfatizar los impactos positivos del turismo y eliminar, en particular, los impactos negativos a nivel económico, social y ambiental, siendo preciso equilibrar la sostenibilidad y el desarrollo turístico del destino. La sostenibilidad del destino turístico se ha convertido, de hecho, en un elemento de diferenciación clave para incrementar su competitividad.

En primer lugar, para la empresa hotelera el medio ambiente y su conservación son aspectos vitales; y de ahí que han surgido iniciativas y propuestas dirigidas a implantar un nuevo concepto relacionado con el turismo, denominado "turismo sostenible" -definido por la O.M.T como "el turismo que tenga plenamente en cuenta sus impactos económicos, sociales y ambientales actuales y futuros, las necesidades de los visitantes, la industria, el medio ambiente y las comunidades de acogida" (O.M.T., 2016b). El entorno es de hecho consustancial al servicio turístico, siendo necesario concienciar a los turistas, residentes y a los gerentes de los alojamientos hoteleros de hacer sostenible el desarrollo de la industria turística con la adopción de prácticas sostenibles ( $c f$. Sanagustín, Monseñe y Gómez, 2011; Vargas, Vaca y García de Soto, 2004).

En segundo lugar, son diversos los autores que resaltan el papel que representa la Responsabilidad Social Empresarial (en adelante, RSE) en el sector turístico. Autores como Wojtarowski et al. (2016) conceptualizan la RSE como pieza clave para el desarrollo sostenible en el sector turístico - y es definida por World Business Council for Sustainable Development (WBCSD) como "el continuo compromiso de las empresas a comportarse éticamente y contribuir al desarrollo económico, mejorar la calidad de vida de los trabajadores y sus familias, así como de la comunidad local, y la sociedad en general".

En tercer lugar, destacada la relevancia del turismo sostenible y la RSE, esta investigación pretende evidenciar la relación entre RSE, Sostenibilidad y Turismo Sostenible. Con ello se pretende también suplir la carencia detectada en la literatura al haberse abordado tradicionalmente el estudio de la RSE desde la óptica del consumidor turístico analizando aspectos tales como, los efectos de la comunicación y difusión de las políticas de RSE implementadas (Alvarado, 2008; Martínez, Pérez y Rodríguez del Bosque, 2014); el nivel de satisfacción y lealtad (Martínez García de Leaniz, 2015; Montaño Valle, 2015; 
Prud'homme y Raymond, 2013); los condicionantes de la adopción de comportamientos responsables y sostenibles por el individuo (Oliveira, Oliveira y Caldeira, 2015; Tilikidou, Delistavrou y Sapountzis, 2014); entre otros.

En consecuencia, el objetivo de esta investigación reside en identificar las actitudes e intenciones conductuales del gerente de los alojamientos hacia la RSE como vía para la implementación de acciones turísticamente sostenibles. En particular, la contextualización de este trabajo se circunscribe al ámbito del medio rural dado el impacto que la actividad turística -especialmente la hotelera- puede representar en dicho entorno. Según Wojtarowski et al. (2016) el desarrollo de acciones turístico responsables en pequeños núcleos rurales puede derivar en impactos positivos y en un desarrollo más sostenible.

Esta investigación asume la Teoría del Desarrollo Sostenible, y define la RSE como un constructo de carácter multidimensional formado por una dimensión económica, social y medioambiental; las cuales son desagregadas por Panwar et al. (2006) y Van Marrewijk (2003), y fundamentadas en el modelo de la "triple bottom line" (en adelante, T.B.L., o "triple cuenta de resultados"; $c f$. Elkington, 1998). En el ámbito turístico ha sido aplicado como referencia teórica en recientes trabajos asociados al contexto hotelero (Alvarado, 2008; Martínez García de Leaniz, 2015; Martínez, Pérez y Rodríguez del Bosque, 2014; Montaño Valle, 2015; Peña y Sierra, 2012; Prud'homme y Raymond, 2013). Por otra parte, atendiendo a la identificación de factores determinantes de la adopción de actitudes socialmente responsables por el gerente rural que deriven en la implantación de estrategias socialmente responsables, esta investigación adopta el marco teórico que proporciona la Teoría del Comportamiento Planeado (en adelante, TCP; $c f$. Ajzen, 1991). TCP es un marco teórico validado y contrastado en una amplia gama de contextos, disciplinas y países incluyendo el ámbito del sector turístico (Gao, Mattila y Lee, 2016; Quintal, Lee y Soutar, 2010). Por ejemplo, Sharma y Sharma (2011) plantean un marco conceptual que relaciona la TCP y la adopción de una estrategia medioambiental proactiva por parte de las empresas familiares, resaltando estos autores la necesidad de evaluar empíricamente dicha relación.

En definitiva, siguiendo las propuestas de Han, Shu y Sheu (2010), Plaza et al. (2012), Yacob y Moorthy (2012) o Yacob et al. (2013) se analiza y evalúa la relación entre la TCP de Ajzen (1991) y la Teoría del Desarrollo Sostenible como instrumento para determinar los factores o variables que inciden en la adopción de cada una de las dimensiones que conforman un comportamiento socialmente responsable en los alojamientos rurales. Es decir, se pretende dar un paso más y determinar si la TCP permite predecir intenciones y conductas socialmente responsables de acuerdo con la Teoría del Desarrollo Sostenible.

El artículo se estructura del siguiente modo. En primer lugar, se presentan los fundamentos teóricos que avalan las hipótesis planteadas en el modelo estructural. En segundo lugar, se describe la metodología empleada para la validación y contrastación de las hipótesis propuestas. Y finalmente, se presentan los resultados y la discusión de las contribuciones de nuestro objeto de estudio.

\section{FUNDAMENTOS TEÓRICOS E HIPÓTESIS DE INVESTIGACIÓN}

El estudio de las actitudes y comportamientos de los directivos se presenta como un elemento clave en el desarrollo de prácticas socialmente responsables (Bennington 
y Minutolo, 2013; Sharma y Sharma, 2011). En este sentido, resulta de especial interés determinar las actitudes empresariales que imperan hacia la RSE con el objeto de identificar y prever cuáles son las tendencias futuras relacionadas con el desarrollo de acciones o políticas en materia de sostenibilidad.

Una de las teorías más destacadas para predecir y comprender el comportamiento social de un individuo procede del ámbito de la psicología social. Concretamente, la TCP de Ajzen (1991) describe el comportamiento de los individuos como una conducta planeada o planificada. El objetivo de esta teoría radica en predecir y explicar la conducta humana partiendo de dos premisas básicas; en primer lugar, que las personas nos comportamos de forma racional, es decir, tenemos en cuenta la información disponible y evaluamos los resultados que originará la realización o no de una acción determinada; y, en segundo lugar, que las acciones están determinadas por la intención del sujeto de llevarlas a cabo, encontrándose bajo el control voluntario de la persona. En general, los actos humanos son frecuentemente racionales; de modo que antes de decidir si se adopta un determinado comportamiento, las personas evalúan las consecuencias de tales acciones, asumiendo por ello un control de la voluntad del individuo. El modelo estructural propuesto evalúa la incidencia de los constructos Actitudes (AC), Normas Subjetivas (NSUB), Control Conductual Percibido (CCP) e Intención Conductual (IC) definidos por la TCP de Ajzen (1991) en el desarrollo de comportamientos sostenibles por parte del gerente del alojamiento turístico rural.

El primer concepto, las actitudes, está constituido a partir de las creencias que posee un individuo acerca de las consecuencias de realizar una determinada conducta, y por la evaluación realizada por el sujeto sobre el resultado de dicha acción. Dichas actitudes determinan las intenciones de las personas a adoptar comportamientos responsables, e.g., la intención de visitar un hotel sostenible (Han, Shu y Sheu, 2010; Han y Kim, 2010; Teng, Wu y Liu, 2015); del directivo empresarial hacia la adopción de medidas responsables dirigidas a incrementar la satisfacción de sus grupos de interés (Plaza et al., 2012); la implementación de comportamientos responsables o medidas concretas tales como la contratación de personas con discapacidad (Jasper y Waldhart, 2013), o la adopción de prácticas medioambientales en su establecimiento (Uhlaner et al., 2012; Yacob y Moorthy, 2012; Yacob et al., 2013), entre otras.

El segundo constructo, NSUB está determinado por la probabilidad de que la conducta a realizar resulte o no aceptable por las personas cuya opinión es determinante para el individuo, así como por la disposición de la persona para adaptarse a las directrices de sus referentes más cercanos. En este sentido, la Teoría Institucional de la RSE postula que el ambiente institucional de la empresa determina las políticas de RSE iniciadas, estando sometida a una presión coercitiva (conocimiento y aplicación de la legislación), una presión normativa (obligación moral, normas sociales) y una presión mimética (imitación o experiencias a seguir) (Montaño Valle, 2015; Sánchez Fernández, 2014). Por tanto, es razonable considerar el papel de las NUSB como antecedente de la IC del individuo, esto es, la influencia social que ejerce el entorno del individuo (e.g., amigos, familiares, compañeros, etc.) sobre la realización de una conducta (Chou, Chen y Wang, 2012; Hong Tan, 2013; Jasper y Waldhart, 2013; Uhlaner et al., 2012; Yacob y Moorthy, 2012; Yacob et al., 2013; Zientara, Kujawski y Bohdanowicz, 2015). 
El tercer constructo, el CCP se refiere a la percepción del individuo de la dificultad o facilidad para desarrollar una conducta o comportamiento (Ajzen, 1991). Se define aquí como la capacidad que el gerente de un alojamiento rural considera que ostenta para desarrollar con éxito unas prácticas socialmente responsables en su establecimiento. A mayor CCP por el individuo, mayor será su IC de adoptar o implantar una conducta o un comportamiento real (Chou, Chen y Wang, 2012; Jasper y Waldhart, 2013; Plaza et al., 2012; Uhlaner et al., 2012; Zientara, Kujawski y Bohdanowicz, 2015). Hong Tan (2013) evidencia que a medida que un individuo dispone de unas mayores creencias de control y percibe que cuenta con unos mayores recursos y capacidades para desarrollar el comportamiento, este lo realizará. En línea con las conclusiones de autores como Yacob y Moorthy (2012) y Yacob et al. (2013) esta investigación propone su inclusión en el análisis del comportamiento del gerente rural.

Por último, el constructo IC determina la intención de llevar a cabo un comportamiento estando conceptualizado como el determinante inmediato de la acción. En línea con las propuestas de Stahl y Sully de Luque (2014) se propone que la intención para desarrollar un comportamiento responsable es influida por factores diferentes a los que determinan un comportamiento real. En nuestro modelo, la IC se formaliza por un constructo de segundo orden de carácter formativo no observable directamente y constituido, según la TCP de Ajzen (1991), por tres constructos: las actitudes del gerente rural hacia la RSE, las NSUB o presión social que recibe el gerente, y el CCP que posee para poder acometer la conducta en cuestión (Figura 1). A pesar de que los constructos formativos de la IC del sujeto pueden no predecir perfectamente el comportamiento del mismo, la literatura científica sugiere que la predicción del comportamiento a través de las intenciones es "el modo más eficiente y simple para realizarlo" (Fishbein y Ajzen, 1975), estableciéndose en recientes trabajos su idoneidad para evaluar comportamientos sociales (Chatzidakis, Kastanakis y Stathopoulou, 2016; Chen y Tung, 2014; Chou, Chen y Wang, 2012; Jasper y Waldhart, 2013; Oliveira, Oliveira y Caldeira, 2015; Teng, Wu y Liu, 2015; Uhlaner et al., 2012; Yacob y Moorthy, 2012; Yacob et al., 2013; Zientara, Kujawski y Bohdanowicz, 2015).

En suma, la robustez e idoneidad que proporciona la TCP (Ajzen, 1991) para la predicción de un comportamiento o de una conducta concreta conduce a su aplicación en campos de estudio o investigaciones muy diversas. En particular, en el ámbito del turismo la aplicación de la TCP de Ajzen (1991), como marco teórico para explicar las actitudes del gerente o directivo turístico hacia acciones socialmente responsables o el comportamiento del consumidor turístico está presente en trabajos tales como los propuestos por Chou, Chen y Wang (2012); Jasper y Waldhart (2013); Uhlaner et al., (2012) o Zientara, Kujawski y Bohdanowicz (2015).

En consecuencia, teniendo en cuenta los planteamientos teóricos expuestos anteriormente, se enuncian las siguientes hipótesis:

$\mathrm{H}_{1}$ : Las actitudes del gerente hacia la RSE influyen positivamente en la intención conductual hacia la implantación de prácticas socialmente sostenibles en el alojamiento rural. 
$\mathrm{H}_{2}$ : Las normas subjetivas o presión social recibida por el gerente influyen positivamente en la intención conductual de implantación de unas prácticas socialmente sostenibles en el alojamiento rural.

$\mathrm{H}_{3}$ : El control conductual percibido por el gerente influye positivamente en la intención conductual de implantación de unas prácticas socialmente sostenibles en el alojamiento rural.

$\mathrm{H}_{4}$ : La intención conductual del gerente de alojamientos rurales sobre la RSE influye positivamente en el desarrollo de un comportamiento turístico sostenible en el establecimiento rural.

\section{Figura 1}

MODELO ESTRUCTURAL DE LA INTENCIÓN CONDUCTUAL DEL GERENTE DE ALOJAMIENTOS RURALES HACIA LA ADOPCIÓN DE COMPORTAMIENTO SOCIALMENTE SOSTENIBLE

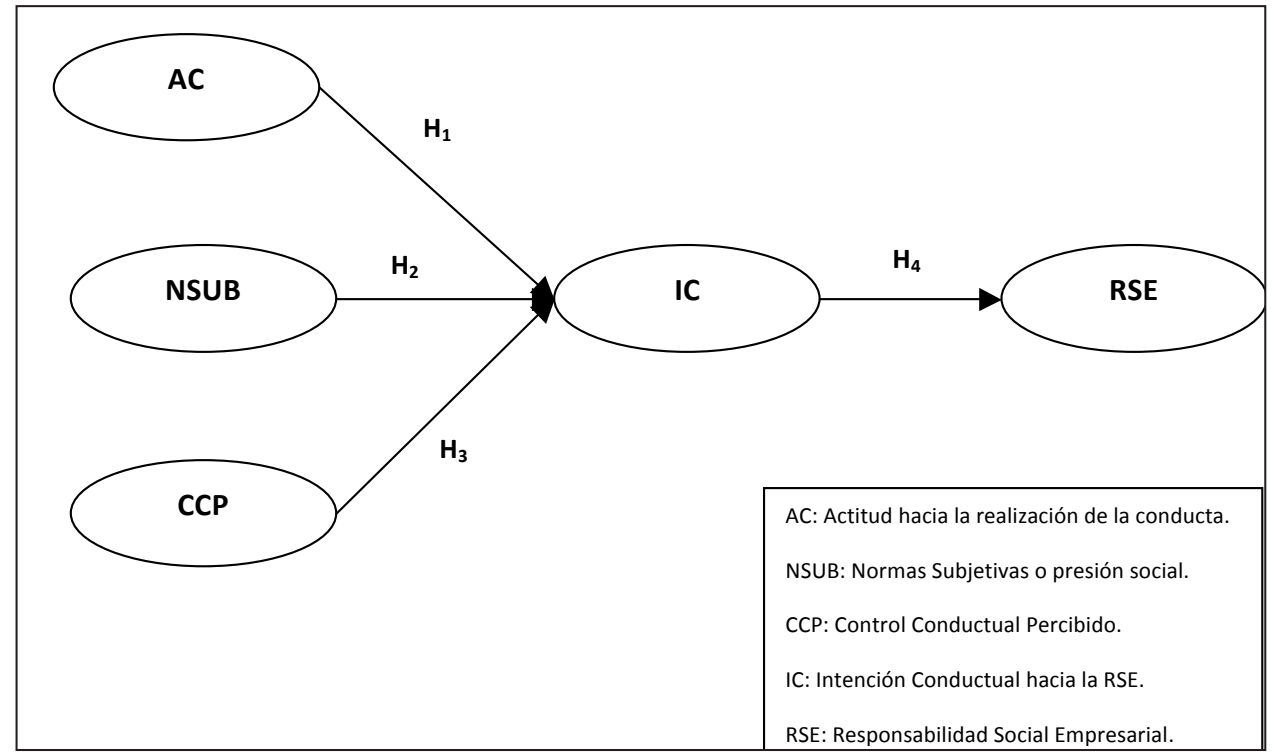

Fuente: Adaptación (Yacob y Moorthy, 2012; Yacob et al., 2013).

\section{METODOLOGÍA}

\subsection{Análisis de datos}

Con el objeto de contrastar empíricamente las hipótesis propuestas se han empleado los modelos de ecuaciones estructurales (Structural equation models, SEM) y, más concretamente, la técnica de mínimos cuadrados parciales (Partial Least Square, PLS). El modelo propuesto pretende, en particular, evaluar la utilidad de los constructos que conforman 
la TCP de Ajzen (1991) como medio para explicar la adopción de un comportamiento socialmente responsable por parte de los gerentes de los alojamientos rurales, atendiendo a las dimensiones definidas en la Teoría del Desarrollo Sostenible (i.e., económica, social y medioambiental). Se han definido cinco constructos: Actitudes (AC), Normas Subjetivas (NSUB), Control Conductual Percibido (CCP), Intención Conductual (IC) y Responsabilidad Social Empresarial (RSE).

Los modelos SEM son aceptados en el campo de la economía de empresa debido a que conjugan, por un lado, la perspectiva psicométrica que modela variables latentes (conceptos no observados directamente) inferidas indirectamente a través de múltiples medidas observadas (indicadores o variables manifiestas) y, por otro, una perspectiva econométrica enfocada a la predicción. Es decir, medimos variables latentes (no observadas) que representan conceptos teóricos, y datos que provienen de medidas (indicadores o variables manifiestas) utilizadas como inputs de análisis estadístico que proporciona evidencia acerca de las relaciones entre variables latentes (Chin, 1998; Williams, Vandenberg y Edwards, 2009).

En este sentido, la justificación de la elección de PLS dirigida a la predicción de las variables dependientes se basa en el carácter exploratorio del trabajo. Más aún, el empleo de PLS conlleva una serie ventajas respecto al tamaño de la muestra o los requisitos de las escalas de medida, lo que permite estimar modelos estructurales con muestras pequeñas, así como con medidas reflectivas y formativas sin problemas de identificación, entre otras ventajas (Hair et al., 2014; Roldán y Sánchez Franco, 2012; Sarstedt, Ringle y Hair, 2014; Sarstedt et al., 2016).

En el análisis del PLS, se emplea el software informático SmartPLS v.3.2.4, mientras que para el análisis descriptivo de los datos se utiliza el programa estadístico IBM SPSS Statistics Version 22.

\section{Figura 2 \\ MODELO PROPUESTO CON CONSTRUCTO DE SEGUNDO ORDEN}

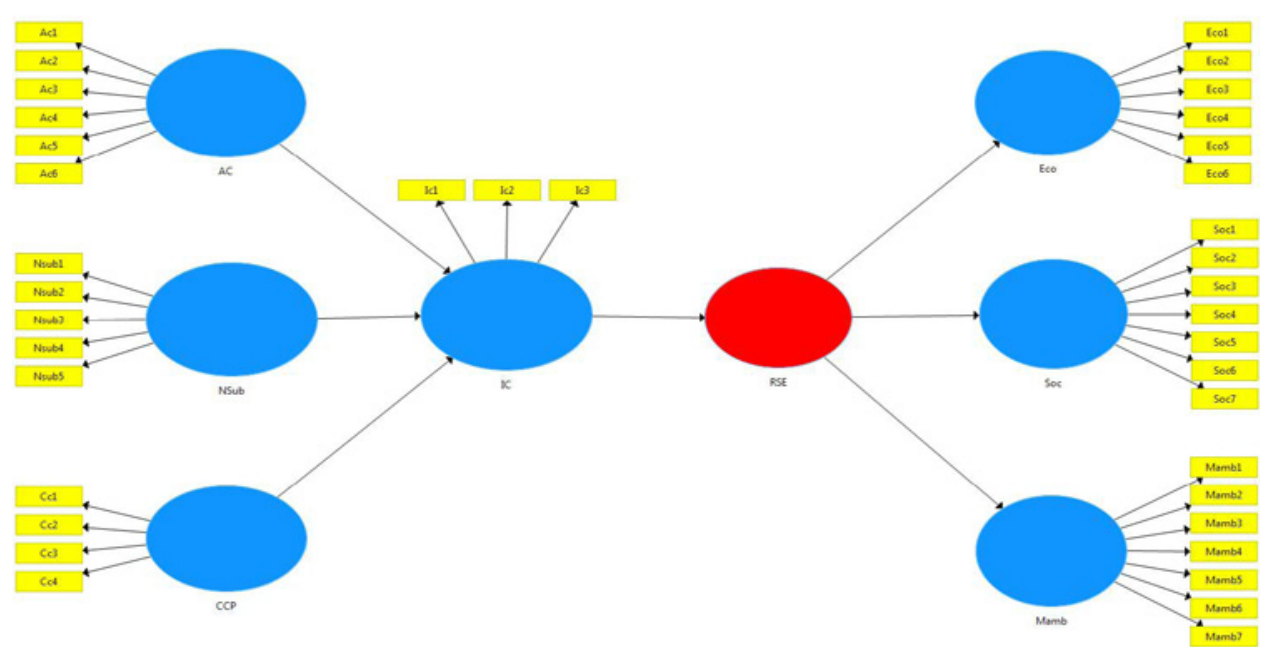




\subsection{Participantes}

Son objeto de esta investigación aquellos establecimientos y apartamentos hoteleros rurales, así como las casas rurales; conforman el universo poblacional 2.708 alojamientos rurales, según los datos facilitados en marzo de 2016 por el Registro Turístico de Andalucía dependiente de la Consejería de Turismo y Comercio de la Junta de Andalucía (España).

Para facilitar la recogida de los datos, el cuestionario se suministró vía online a una muestra formada por 1.578 alojamientos rurales, de los cuales 303 ostentan la categoría de hotel rural, 73 hostales rurales, 69 pensiones rurales, 178 apartamentos rurales y 955 casas rurales. Se obtienen una tasa de respuesta del 16\%, es decir, 252 respuestas válidas entre los meses de mayo y junio de 2016.

La edad media del encuestado corresponde a 47,6 años (desviación típica: 20,5 años) representando los hombres el 59,5\%. El perfil general del encuestado es el responsable o gerente de dichos alojamientos rurales. El 84,5\% de los encuestados afirman desempeñar labores de administración, gestión o propiedad en el alojamiento rural, lo que contribuye a la contrastación de las hipótesis formuladas.

\section{Tabla 1 \\ FICHA TÉCNICA DEL ESTUDIO EMPÍRICO}

\begin{tabular}{l|l}
\hline Universo & $\begin{array}{l}\text { Establecimientos hoteleros y apartamentos turísticos en su modalidad } \\
\text { hotelera (hotel, hotel-apartamento rural y pensión) y modalidad no } \\
\text { hotelera: apartamento rural; y casas rurales. }\end{array}$ \\
\hline Ámbito & Andalucía (España). \\
\hline $\begin{array}{l}\text { Método de recogida } \\
\text { de información }\end{array}$ & Encuesta online. \\
\hline Base de datos & $\begin{array}{l}\text { Elaboración propia a partir de los datos facilitados por el Registro de } \\
\text { Turismo de Andalucía (España). }\end{array}$ \\
\hline Unidad muestral & $\begin{array}{l}\text { Establecimientos hoteleros y apartamentos turísticos en su modalidad } \\
\text { hotelera (hotel, hotel-apartamento rural y pensión) y modalidad no } \\
\text { hotelera: apartamento rural; y casas rurales de Andalucía. }\end{array}$ \\
\hline Trabajo de campo & Fecha entre el 16 de mayo y el 15 de junio de 2016. \\
\hline Número de encuestas & 261 \\
\hline Respuestas válidas & 252 \\
\hline
\end{tabular}

\subsection{Medidas}

La validez de contenido consiste en determinar si la escala empleada refleja adecuadamente las dimensiones del constructo que se pretenden estimar con la misma. En este sentido, la revisión de la literatura y la realización durante el mes de mayo de 2016 de un pretest del cuestionario inicial por un grupo de seis expertos compuesto tanto por académicos como profesionales del sector turístico, ha permitido identificar los ítems más relevantes para los constructos objeto de medición. 
Los constructos se miden empleando escalas propuestas y validadas en los trabajos revisados. En particular, se emplean 18 ítems que evalúan los constructos AC (6 ítems), NSUB (5 ítems), CCP (4 ítems) e IC (3 ítems) para el desarrollo de un comportamiento turístico sostenible por parte del gerente rural (Chatzidakis, Kastanakis y Stathopoulou, 2016; Chou, Chen y Wang, 2012; Garay y Font, 2013; Hong Tan, 2013; Yacob et al., 2013, entre otros). Mientras que para la medición del constructo RSE se emplea una escala compuesta por 21 indicadores, para evaluar la dimensión económica (6 ítems); la dimensión social (7 ítems) y la dimensión medioambiental (7 ítems) que conforman este constructo (Font, Garay y Jones, 2014; Garay y Font, 2013; Vaca Acosta, 2013, entre otros).

La escala de medición elegida, en todos los casos, es de tipo Likert: 1, muy en desacuerdo, a 7 , muy de acuerdo.

\section{RESULTADOS}

El empleo de la técnica PLS implica, por un lado, la evaluación o estimación del modelo de medida cuyo objetivo principal radica en determinar que cada una de las escalas utilizadas mida exclusivamente el constructo que se pretende estimar; y por otro lado, la evaluación de las relaciones estructurales entre los diferentes constructos que conforman el modelo propuesto (Hair, Ringle y Sarstedt, 2011; Roldan y Sánchez Franco, 2012).

El modelo propuesto presenta la peculiaridad de la existencia de un constructo de segundo orden -Responsabilidad Social Empresarial (RSE)- que no está medido por ninguna variable manifiesta o indicador, lo que implica necesariamente la realización de un tratamiento previo para poder evaluar el modelo de medida y el modelo estructural. En este sentido, se ha empleado el enfoque de dos pasos propuesto por Wright et al., (2012), teniendo en consideración las recomendaciones realizadas por Ciavolino y Nitti (2013) que sugieren adoptar este enfoque cuando el objeto de la investigación es la medición de un constructo con un alto nivel de abstracción, así como debido a que no existe el mismo número de indicadores para cada constructo (Chin, 1998).

\subsection{Modelo de medida de primer orden}

En la evaluación del modelo propuesto y dada la existencia de un constructo de segundo orden se aplica la primera etapa del método de dos pasos propuesto por Wright et al., (2012), en el que los factores de primer orden van a actuar en el modelo como el constructo de segundo orden al que representan.

La fiabilidad individual de cada ítem del modelo de medida es evaluada a través de sus cargas factoriales $(\lambda)$.

A nivel general se establece que un estimador o indicador es aceptado cuando posee con su constructo una carga igual o superior a 0,707 (Barclay, Higgins y Thompson, 1995; Hair, Ringle y Sarstedt, 2011). No obstante, recientes trabajos establecen como aceptables niveles superiores a 0,4 en etapas iniciales de desarrollo de escalas o por la novedad del tema tratado (Richter et al., 2016). En nuestro caso, se procede pues a la depuración de la escala o eliminación de aquellos indicadores cuyas cargas factoriales sean inferiores a 0,4. Asimismo, se evalúan las cargas cruzadas (cross loadings) para determinar en qué 
medida los indicadores miden exclusivamente el constructo al que se encuentran referidos o bien contribuyen en mayor grado a la determinación de otro constructo. En suma, tras la eliminación de los ítems Cc2, Cc4, Eco5, Ac4, Mamb3, Ac6 y Soc7 se obtiene unas cargas factoriales $(\lambda)$ superiores a 0,50 (Hair et al., 2014) (Tabla 2).

Tabla 2

MATRIZ DE CARGAS FACTORIALES (A) Y CARGAS CRUZADAS DESPUÉS DE LA DEPURACIÓN DE ÍTEMS

\begin{tabular}{cccccccc}
\hline & AC & CCP & ECO & IC & MAMB & NSUB & SOC \\
\hline Ac1 & $\mathbf{0 , 6 5 4}$ & 0,079 & 0,258 & 0,209 & 0,286 & 0,137 & 0,308 \\
Ac2 & $\mathbf{0 , 8 1 4}$ & 0,002 & 0,274 & 0,328 & 0,307 & 0,329 & 0,427 \\
Ac3 & $\mathbf{0 , 6 7 5}$ & 0,135 & 0,244 & 0,236 & 0,204 & 0,348 & 0,257 \\
Ac5 & $\mathbf{0 , 7 5 5}$ & 0,043 & 0,415 & 0,391 & 0,341 & 0,557 & 0,331 \\
\hline Cc1 & 0,091 & $\mathbf{0 , 9 8 0}$ & 0,009 & 0,175 & 0,081 & 0,189 & 0,103 \\
Cc3 & $-0,018$ & $\mathbf{0 , 6 0 9}$ & 0,056 & 0,044 & 0,051 & 0,075 & $-0,061$ \\
\hline Eco1 & 0,338 & 0,069 & $\mathbf{0 , 6 8 9}$ & 0,317 & 0,397 & 0,170 & 0,396 \\
Eco2 & 0,255 & 0,003 & $\mathbf{0 , 5 2 0}$ & 0,189 & 0,206 & 0,385 & 0,226 \\
Eco3 & 0,273 & $-0,097$ & $\mathbf{0 , 8 0 0}$ & 0,347 & 0,472 & 0,275 & 0,386 \\
Eco4 & 0,220 & 0,026 & $\mathbf{0 , 6 7 3}$ & 0,225 & 0,332 & 0,234 & 0,307 \\
Eco6 & 0,305 & 0,097 & $\mathbf{0 , 5 5 3}$ & 0,237 & 0,399 & 0,241 & 0,264 \\
\hline Ic1 & 0,334 & 0,131 & 0,354 & $\mathbf{0 , 9 0 5}$ & 0,416 & 0,422 & 0,453 \\
Ic2 & 0,453 & 0,189 & 0,380 & $\mathbf{0 , 8 4 0}$ & 0,425 & 0,487 & 0,390 \\
Ic3 & 0,279 & 0,095 & 0,329 & $\mathbf{0 , 8 2 9}$ & 0,332 & 0,383 & 0,403 \\
\hline Mamb1 & 0,365 & 0,036 & 0,451 & 0,405 & $\mathbf{0 , 7 8 4}$ & 0,270 & 0,450 \\
Mamb2 & 0,204 & 0,099 & 0,377 & 0,237 & $\mathbf{0 , 6 7 8}$ & 0,197 & 0,321 \\
Mamb4 & 0,234 & 0,075 & 0,312 & 0,166 & $\mathbf{0 , 5 9 4}$ & 0,085 & 0,223 \\
Mamb5 & 0,339 & 0,045 & 0,459 & 0,315 & $\mathbf{0 , 7 7 9}$ & 0,253 & 0,449 \\
Mamb6 & 0,348 & 0,027 & 0,474 & 0,451 & $\mathbf{0 , 8 4 7}$ & 0,363 & 0,530 \\
Mamb7 & 0,282 & 0,127 & 0,476 & 0,386 & $\mathbf{0 , 8 3 0}$ & 0,301 & 0,473 \\
\hline Nsub1 & 0,443 & 0,102 & 0,332 & 0,410 & 0,209 & $\mathbf{0 , 8 4 7}$ & 0,362 \\
Nsub2 & 0,421 & 0,146 & 0,297 & 0,411 & 0,164 & $\mathbf{0 , 8 3 8}$ & 0,290 \\
Nsub3 & 0,207 & 0,057 & 0,202 & 0,172 & 0,166 & $\mathbf{0 , 6 0 0}$ & 0,156 \\
Nsub4 & 0,415 & 0,185 & 0,275 & 0,380 & 0,375 & $\mathbf{0 , 6 9 4}$ & 0,359 \\
Nsub5 & 0,372 & 0,170 & 0,301 & 0,445 & 0,369 & $\mathbf{0 , 7 7 8}$ & 0,364 \\
\hline Soc1 & 0,299 & 0,014 & 0,344 & 0,330 & 0,323 & 0,226 & $\mathbf{0 , 6 0 1}$ \\
Soc2 & 0,387 & 0,005 & 0,390 & 0,395 & 0,523 & 0,368 & $\mathbf{0 , 8 0 4}$ \\
Soc3 & 0,414 & 0,074 & 0,365 & 0,307 & 0,349 & 0,355 & $\mathbf{0 , 6 6 5}$ \\
Soc4 & 0,359 & 0,173 & 0,366 & 0,378 & 0,420 & 0,351 & $\mathbf{0 , 7 9 0}$ \\
Soc5 & 0,141 & 0,132 & 0,267 & 0,263 & 0,336 & 0,183 & $\mathbf{0 , 6 1 3}$ \\
Soc6 & 0,300 & $-0,056$ & 0,340 & 0,343 & 0,393 & 0,266 & $\mathbf{0 , 7 1 1}$ \\
\hline & & & & & & & \\
\hline
\end{tabular}

La evaluación de la fiabilidad del constructo o consistencia interna se evaluó empleando el coeficiente de fiabilidad compuesta $\left(\rho_{\mathrm{c}}\right)$ (composity realibity). Su evaluación permite 
comprobar el grado de rigurosidad con el que las variables manifiestas o indicadores miden la misma variable latente. Los valores de la fiabilidad compuesta considerados como satisfactorios han de estar entre valores de 0,60 y 0,70 en estudios exploratorios; y de forma más estricta en investigaciones más avanzadas entre 0,70 y 0,90 (Nunnally y Bernstein, 1994); mientras que niveles por debajo de 0,60 indicarían una falta de fiabilidad (Hair, Ringle y Sarstedt, 2011; Richter et al., 2016).

En la Tabla 3 se ofrece el valor de la fiabilidad compuesta antes y después de la depuración de ítems. En nuestro estudio, los constructos latentes son fiables al presentar medidas de consistencia interna $\left(\rho_{\mathrm{c}}\right)$ que exceden el valor de 0,7 .

Tabla 3

MATRIZ DE FIABILIDAD Y VALIDEZ DE CONSTRUCTO DEL MODELO DE PRIMER ORDEN

\begin{tabular}{l|c|c|c|c}
\hline \multirow{2}{*}{} & \multicolumn{2}{|c|}{ Antes depuración ítems } & \multicolumn{2}{c}{ Después de la depuración de ítems } \\
\cline { 2 - 5 } & $\begin{array}{c}\text { Fiabilidad } \\
\text { compuesta }\left(\rho_{\mathrm{c}}\right)\end{array}$ & $\begin{array}{c}\text { Varianza extraída } \\
\text { media (AVE) }\end{array}$ & $\begin{array}{c}\text { Fiabilidad } \\
\text { compuesta }\left(\rho_{\mathrm{c}}\right)\end{array}$ & $\begin{array}{c}\text { Varianza extraída } \\
\text { media (AVE) }\end{array}$ \\
\hline AC & 0,791 & 0,409 & 0,817 & 0,529 \\
CCP & 0,259 & 0,234 & 0,791 & 0,666 \\
ECO & 0,760 & 0,366 & 0,786 & 0,429 \\
IC & 0,894 & 0,737 & 0,894 & 0,738 \\
MAMB & 0,873 & 0,509 & 0,888 & 0,573 \\
NSUB & 0,868 & 0,573 & 0,868 & 0,573 \\
SOC & 0,851 & 0,454 & 0,852 & 0,493 \\
\hline
\end{tabular}

En segundo lugar, se evalúa la validez convergente. Esta implica determinar si un conjunto de indicadores representan o miden un único constructo subyacente, pudiendo ello ser demostrado por medio de su unidimensionalidad (Henseler, Ringle y Sinkovics, 2009). Se valora a través del empleo de varianza extraída media (Average Variance Extracted, en adelante AVE). Un valor AVE superior a 0,50 indica pues que existe un grado adecuado de validez convergente, es decir, más de la mitad de la varianza de la variable latente es explicada por sus indicadores (Hair, Ringle y Sarstedt, 2011; Hair et al., 2014). Las varianzas extraídas medias de nuestros constructos superan los valores 0,5 excepto para las dimensiones económica y social del constructo RSE cuyos valores se encuentran muy próximos al nivel crítico.

En tercer lugar, la evaluación de la validez discriminante determina en qué medida un constructo concreto es diferente de otros constructos con el objeto de detectar posibles problemas de solapamiento, es decir, el constructo debe compartir más varianza con sus variables manifiestas o indicadores que con otros constructos del modelo (Barclay, Higgins y Thompson, 1995). Para proceder a este análisis se emplean tres procedimientos. El primero de ellos, el criterio de Fornell-Larcker (1981) considera que el constructo latente comparte más varianza con sus indicadores asignados que con otra variable latente en el modelo. En 
términos estadísticos el AVE de cada constructo latente debería ser mayor que la varianza que dicho constructo comparte con los otros constructos del modelo (Barclay, Higgins y Thompson, 1995; Hair, Ringle y Sarstedt, 2011; Henseler, Ringle y Sinkovics, 2009; Richter et al., 2016). En este sentido, una forma equivalente de hacer este análisis es demostrar que las correlaciones entre los constructos son más bajas que la raíz cuadrada del AVE (Tabla 4).

\section{Tabla 4}

VALIDEZ DISCRIMINANTE DE LOS CONSTRUCTOS DE PRIMER ORDEN: CRITERIO DE FORNELL-LARCKER

\begin{tabular}{lccccccc}
\hline & AC & CCP & ECO & IC & MAMB & NSUB & SOC \\
\hline AC & $\mathbf{0 , 7 2 7}$ & & & & & & \\
CCP & 0,076 & $\mathbf{0 , 8 1 6}$ & & & & & \\
ECO & 0,424 & 0,020 & $\mathbf{0 , 6 5 5}$ & & & & \\
IC & 0,420 & 0,164 & 0,414 & $\mathbf{0 , 8 5 9}$ & & & \\
MAMB & 0,398 & 0,083 & 0,568 & 0,459 & $\mathbf{0 , 7 5 7}$ & & \\
NSUB & 0,508 & 0,183 & 0,378 & 0,505 & 0,348 & $\mathbf{0 , 7 5 7}$ & \\
SOC & 0,460 & 0,077 & 0,495 & 0,484 & 0,563 & 0,422 & $\mathbf{0 , 7 0 2}$ \\
\hline
\end{tabular}

El segundo criterio para determinar la validez discriminante menos exigente o estricto es evaluar las cargas cruzadas (cross loadings) debiéndose cumplir que un indicador cargue o correlacione más sobre su propio constructo que sobre el resto de variables latentes del modelo (Hair, Ringle y Sarstedt, 2011; Henseler, Ringle y Sinkovics, 2009). Esta matriz se evalúa anteriormente durante el proceso de análisis individual de ítems existiendo validez discriminante. Finalmente, el tercer criterio empleado de reciente aplicación es el denominado Heterotrait-Monotrait Ratio (HTMT). Según HTMT existe validez discriminante cuando las correlaciones entre los constructos es inferior a 0,85 (Richter et al., 2016) (Tabla 5). En nuestro caso los constructos evaluados poseen validez discriminante.

Tabla 5

VALIDEZ DISCRIMINANTE DE LOS CONSTRUCTOS DE PRIMER ORDEN: HETEROTRAIT-MONOTRAIT RATIO (HTMT)

\begin{tabular}{lccccccc}
\hline & AC & CCP & ECO & IC & MAMB & NSUB & SOC \\
\hline AC & & & & & & & \\
CCP & 0,157 & & & & & \\
ECO & 0,601 & 0,200 & & & & & \\
IC & 0,515 & 0,182 & 0,545 & & & & \\
MAMB & 0,492 & 0,133 & 0,729 & 0,510 & & & \\
NSUB & 0,595 & 0,222 & 0,543 & 0,583 & 0,382 & & \\
SOC & 0,597 & 0,192 & 0,671 & 0,597 & 0,649 & 0,501 & \\
\hline
\end{tabular}




\subsection{Modelo de medida de segundo orden}

Tras la realización de la etapa anterior, necesaria para evaluar un modelo multidimensional de segundo orden, la segunda etapa consiste en emplear las puntuaciones agregadas para modelar el constructo de segundo orden RSE (Wright et al., 2012). En este momento del proceso el modelo adopta una estructura nomológica diferente; es necesario volver a evaluar el modelo de medida. Se procede pues a analizar la fiabilidad individual de cada ítem determinada a través de sus cargas factoriales $(\lambda)$ que en el caso del constructo de segundo orden RSE son medidas a través de ECO, SOC y MAMB. Se obtienen unas cargas factoriales $(\lambda)$ superiores a 0,60 (Hair et al., 2014).

En cuanto a la fiabilidad compuesta $\left(\varrho_{c}\right)$ se obtienen en todos los casos valores por encima de 0,70, mientras que la validez convergente evaluada a través de AVE presenta valores superiores a 0,50 (Hair, Ringle y Sarstedt, 2011; Hair et al., 2014;) (Tabla 6).

\section{Tabla 6 \\ ANÁLISIS DE LA FIABILIDAD INDIVIDUAL, FIABILIDAD DE CONSTRUCTO Y VALIDEZ CONVERGENTE DEL MODELO DE SEGUNDO ORDEN}

\begin{tabular}{|c|c|c|c|}
\hline & $\begin{array}{l}\text { Carga } \\
\text { factorial } \\
(\lambda)\end{array}$ & $\begin{array}{l}\text { Fiabilidad } \\
\text { compuesta } \\
\left(\rho_{c}\right)\end{array}$ & $\begin{array}{c}\text { Varianza } \\
\text { extraída } \\
\text { media (AVE) }\end{array}$ \\
\hline Actitudes (AC) & - & 0,817 & 0,529 \\
\hline $\begin{array}{l}\text { Ac1: Las prácticas socialmente responsables aportan } \\
\text { beneficios para la sociedad en general. }\end{array}$ & 0,653 & - & - \\
\hline $\begin{array}{l}\text { Ac2: La aplicación de prácticas socialmente } \\
\text { responsables son beneficiosas para mi establecimiento. }\end{array}$ & 0,814 & - & - \\
\hline $\begin{array}{l}\text { Ac3: El gerente socialmente responsable debe } \\
\text { anteponer los intereses de la sociedad sobre los } \\
\text { intereses de la propia empresa. }\end{array}$ & 0,675 & - & - \\
\hline $\begin{array}{l}\text { Ac5: Considero que el desarrollo de acciones } \\
\text { responsables y sostenibles son necesarias para } \\
\text { satisfacer las necesidades de mis clientes. }\end{array}$ & 0,756 & - & - \\
\hline Control Conductual Percibido (CCP) & - & 0,792 & 0,667 \\
\hline $\begin{array}{l}\mathrm{Cc} 1 \text { : Se requiere de un esfuerzo extra de tiempo y } \\
\text { recursos. }\end{array}$ & 0,980 & - & - \\
\hline $\begin{array}{l}\text { Cc3: Existe un alto coste en la certificación y } \\
\text { verificación de las prácticas de responsabilidad social } \\
\text { adoptadas. }\end{array}$ & 0,611 & - & - \\
\hline Intención Conductual (IC) & - & 0,894 & 0,737 \\
\hline $\begin{array}{l}\text { Ic1: Mi empresa tiene previsto mejorar sus estrategias } \\
\text { en materia de responsabilidad social para conseguir un } \\
\text { establecimiento más sostenible. }\end{array}$ & 0,903 & - & - \\
\hline
\end{tabular}




\begin{tabular}{|c|c|c|c|}
\hline $\begin{array}{l}\text { Ic2: Considero que la responsabilidad social es un } \\
\text { aspecto relevante dentro de las estrategias de mi } \\
\text { establecimiento. }\end{array}$ & 0,845 & - & - \\
\hline $\begin{array}{l}\text { Ic3: Mi establecimiento tiene previsto incorporar en } \\
\text { el plazo de un año decisiones más responsables y } \\
\text { sostenibles en su modelo de gestión. }\end{array}$ & 0,826 & - & - \\
\hline Normas Subjetivas (NSUB) & - & 0,868 & 0,573 \\
\hline $\begin{array}{l}\text { Nsub1: Mejorar la imagen de mi establecimiento ante } \\
\text { la sociedad. }\end{array}$ & 0,846 & - & - \\
\hline $\begin{array}{l}\text { Nsub2: Mejorar la posición competitiva de mi } \\
\text { establecimiento. }\end{array}$ & 0,837 & - & - \\
\hline Nsub3: Cumplir las obligaciones legales. & 0,600 & - & - \\
\hline Nsub4: Mejorar la calidad de vida de la comunidad local. & 0,694 & - & - \\
\hline $\begin{array}{l}\text { Nsub5: La preferencia del cliente por alojarse en } \\
\text { un establecimiento sostenible y responsable con su } \\
\text { entorno. }\end{array}$ & 0,778 & - & - \\
\hline Responsabilidad Social Empresarial (RSE) & - & 0,872 & 0,694 \\
\hline Dimensión económica (ECO) & 0,808 & - & - \\
\hline Dimensión social (SOC) & 0,837 & - & - \\
\hline Dimensión Medioambiental (MAMB) & 0,854 & - & - \\
\hline
\end{tabular}

Por último, en cuanto a la validez discriminante también se presentan valores adecuados analizando la matriz de cargas cruzadas y aplicando el criterio de Fornell-Larcker y Heterotrait-Monotrait Ratio (HTMT).

\subsection{Modelo estructural}

Una vez evaluado el modelo de medida, se procede a testar las relaciones estructurales entre las variables hipotetizadas. La bondad de un modelo teórico se determina a través de los niveles de significación de los coeficientes de los caminos o path (path coefficients, $\beta$ ), es decir, la relación entre los constructos y la predictibilidad de los constructos endógenos (variables dependientes). La significación de los caminos se obtiene por medio de la técnica de bootstrapping; mientras que para cada constructo dependiente se calcula también la cantidad de varianza del constructo explicada por el modelo o $\mathrm{R}^{2}$. Si un camino no fuera significativo u ofreciera un signo contrario a la dirección de la hipótesis propuesta implicaría que no se soporta la hipótesis; mientras que si el camino es significativo empíricamente supondría el apoyo de la relación causal planteada. Un valor de $\mathrm{R}^{2}$ de $0,75,0,5$ o 0,25 para las variables endógenas del modelo estructural suele ser descrito como sustanciales, moderados y débiles respectivamente (Hair, Ringle y Sarstedt, 2011).

En nuestro modelo el constructo IC presenta un valor de la varianza explicada igual al $30 \%$. Por otro lado, la RSE presenta también un valor de la varianza explicada igual al $30 \%$ (Tabla 7). 
Tabla 7

EFECTOS DE LAS VARIABLES ENDÓGENAS

\begin{tabular}{lccccc}
\hline \multicolumn{1}{c}{ Hipótesis } & $\mathrm{R}^{2}$ & $\mathrm{Q}^{2}$ & $\begin{array}{c}\text { Efecto directo } \\
(\beta)\end{array}$ & Correlación & $\begin{array}{c}\text { Varianza } \\
\text { explicada }\end{array}$ \\
\hline H1: AC -> IC & & & $0,223 * * *$ & 0,421 & $9,4 \%$ \\
H2: NSUB -> IC & $29,8 \%$ & 0,193 & $0,378^{* * *}$ & 0,506 & $19,1 \%$ \\
H3: CCP -> IC & & & 0,079 ns & 0,165 & $1,3 \%$ \\
H4: IC -> RSE & $29,7 \%$ & 0,199 & $0,545 * * *$ & 0,545 & $29,7 \%$ \\
\hline
\end{tabular}

Más aún, otra medida de evaluación de la capacidad predictiva del modelo estructural es el denominado Test de Stone-Geisser $\left(\mathrm{Q}^{2}\right)$ evaluado a través del procedimiento de blindfolding (Hair, Ringle y Sarstedt, 2011; Richter et al., 2016). Si el valor para cierta variable latente endógena es superior a cero, el constructo latente posee relevancia predictiva (Hair, Ringle y Sarstedt, 2011). Como puede observarse en la tabla anterior, la aplicación de este procedimiento revela que tanto la IC como la RSE son constructos que poseen validez predictiva.

Por último, el análisis de la significación de los coeficientes paths $(\beta)$ o pesos de regresión estandarizados contribuye a verificar en qué medida las variables predictoras contribuyen a la varianza explicada de las variables endógenas, lo que permite corroborar si las hipótesis propuestas son estadísticamente significativas (Hair, Ringle y Sarstedt, 2011). Los resultados se presentan en la Tabla 8 en la que se observa que todas las hipótesis propuestas son soportadas empíricamente, a excepción de la relación entre el CCP y la IC que es estadísticamente no significativa.

Para corroborar los hallazgos anteriores, se aplica una técnica no paramétrica: intervalos de confianza. Según Henseler, Ringle y Sinkovics (2009) si "un intervalo de confianza para un coeficiente path - w - no incluye al valor cero, entonces la hipótesis de que - w es igual a cero se rechaza". Se comprueba que aplicando este procedimiento se confirman los resultados anteriores (Tabla 8).

Tabla 8

\section{RESULTADOS DEL MODELO ESTRUCTURAL}

\begin{tabular}{lcccccc}
\hline \multirow{2}{*}{ Hipótesis } & $\begin{array}{c}\text { Coeficiente } \\
\text { path }(\beta)\end{array}$ & $\begin{array}{c}\text { Estadístico t } \\
\text { (bootstrap) }\end{array}$ & Soportada & \multicolumn{2}{c}{$\begin{array}{c}\text { Intervalo de Confianza } \\
\text { (bootstrap 95\%) }\end{array}$} & Soportada \\
\cline { 1 - 3 } H1: AC -> IC & $0,223 * * *$ & 3,002 & SI & 0,078 & 0,370 & SI \\
H2: NSUB -> IC & $0,378 * * *$ & 5,223 & SI & 0,230 & 0,516 & SI \\
H3: CCP -> IC & 0,079 ns & 1,497 & NO & $-0,029$ & 0,187 & NO \\
H4: IC -> RSE & $0,545 * * *$ & 9,574 & SI & 0,428 & 0,652 & SI \\
\hline
\end{tabular}

Para $\mathrm{n}=5.000$ submuestras: $* \mathrm{p}<0.05 ; * * \mathrm{p}<0.01 ; * * * \mathrm{p}<0.001$ (basado en una distribución $\mathrm{t}$ de Student de una cola). $\mathrm{t}(0,1 ; 4999)=1,645 ; \mathrm{t}(0,05 ; 4999)=1,960 ; \mathrm{t}(0,001 ; 4999)=3,292$. 


\section{Figura 3}

\section{MODELO ESTRUCTURAL}

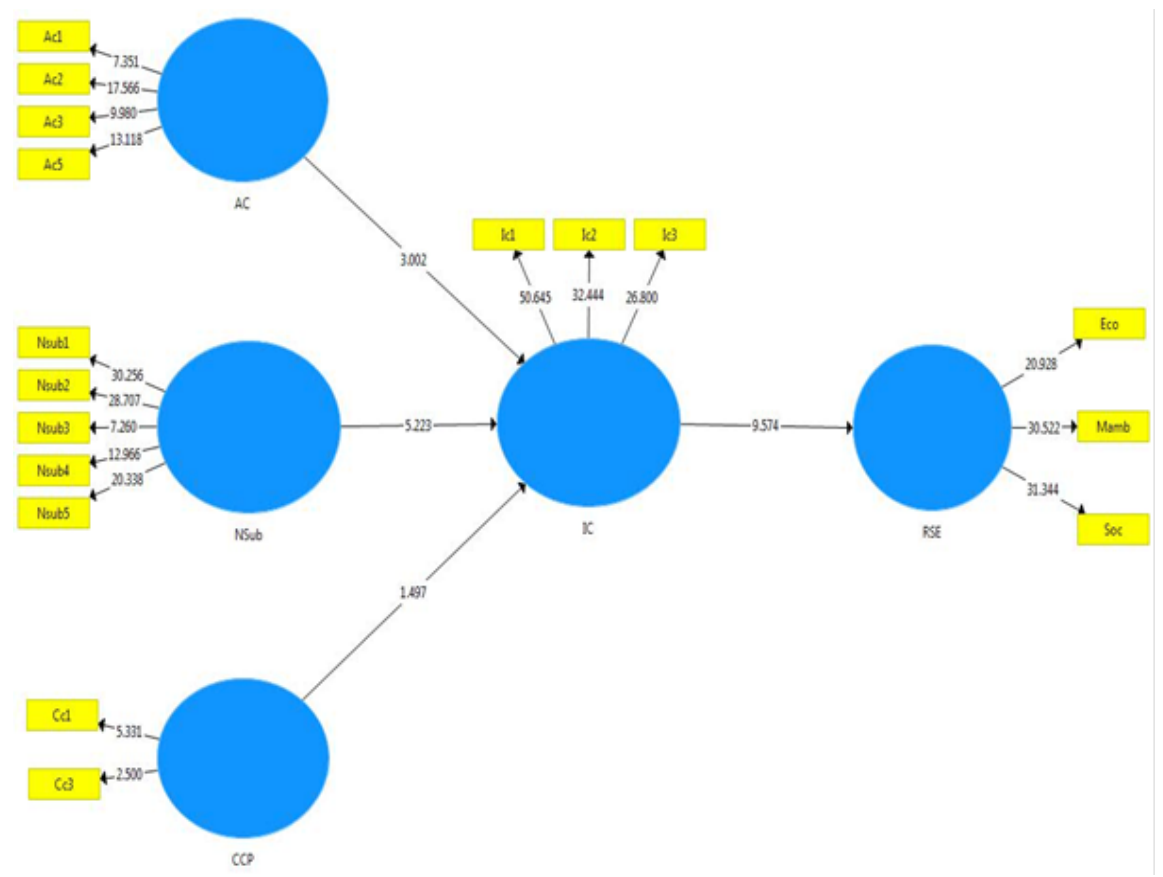

\section{CONCLUSIONES}

La importancia del desarrollo de un auténtico turismo sostenible especialmente en el medio rural, dado el impacto que sobre el mismo puede ocasionar su no adopción (i.e., deterioro del entorno, excesiva afluencia de visitantes, pérdida de tradiciones, etc.), conducen a la necesidad de promover la adopción de unas actitudes socialmente responsables por parte de los gerentes de los alojamientos rurales que deriven en la implementación de estrategias centradas en la sostenibilidad.

En este sentido, destaca el carácter novedoso de este trabajo al testar la relación conceptual entre la TCP de Ajzen (1991) y la Teoría del Desarrollo Sostenible, evidenciándose la relación causal planteada entre AC e IC, y explicando las actitudes el 9,4\% de la IC del gerente. Este resultado puede ser explicado parcialmente por la reducida dimensión empresarial de los alojamientos rurales que da lugar a ciertas debilidades en cuanto a la disposición de los recursos materiales y humanos necesarios para acometer las acciones socialmente responsables en el establecimiento, tal y como sugieren Garay y Font (2013) entre otros.

La implementación de acciones de RSE en los alojamientos rurales está determinada principalmente por cuestiones normativas, legales o influencias del entorno del gerente, que se confirma por medio de la validación empírica de la segunda hipótesis. En particular, el constructo NSUB determina el mayor grado de variabilidad de la IC del gerente hacia 
la adopción de dichas prácticas, representando concretamente un 19,1\%. Las NSUB o la presión social que recibe el gerente es pues el constructo más influyente en la intención de adopción en su establecimiento de medidas socialmente responsables. Es decir, el desarrollo de dichas acciones no se debe a creencias y convicciones sólidas y propias del sujeto, sino que se determinan por su entorno.

Asimismo, entre los factores que determinan la adopción de medidas de RSE se encuentra el nivel de capacidad y control que el individuo considera que posee para acometerlas. No ha sido soportada, sin embargo, la tercera hipótesis del modelo estructural siendo la relación entre el CCP y la IC no significativa. A nuestro juicio, la reducida dimensión empresarial que caracteriza a los alojamientos rurales puede ser un factor que determine que el constructo CCP sea no significativo estadísticamente en la determinación de la IC del gerente, dada la menor capacidad de recursos y medios disponibles de estos establecimientos para acometer acciones socialmente responsables. Asimismo, existe la percepción de que es necesario la realización de un esfuerzo extra de tiempo y recursos en su adopción, lo que corrobora que el control y la capacidad percibida por el individuo sea baja y no explique ni determine la variabilidad de la IC hacia la implementación de acciones socialmente responsables en el alojamiento rural.

En este sentido, se recomienda que los gerentes de los alojamientos rurales realicen un mayor esfuerzo de formación y capacitación que contribuirá a tomar conciencia de los beneficios reales que la adopción de medidas socialmente sostenibles suponen para el establecimiento y para su entorno.

Por último, se confirma el soporte empírico de la cuarta hipótesis formulada, de modo que la IC explica el 29,7\% de la aplicación de las acciones adoptadas en materia de RSE en el alojamiento rural, siendo significativa dicha relación causal. No obstante, cabe advertir que este resultado puede venir condicionado y limitado por la existencia de ciertas percepciones o creencias que llevan al sujeto a neutralizar su intención real de adoptar unas prácticas socialmente responsables, a pesar de la valoración positiva que de ellas realizan, tal y como sugieren trabajos como el realizado por Gruber y Schlegelmilch (2014).

Además, las aparentes actitudes e intenciones positivas hacia la RSE no se traducen o explican en su totalidad el comportamiento real del gerente, pudiendo desarrollarse, en este caso, lo que algunos autores denominan como "sostenibilidad selectiva" al existir una diferencia entre la importancia percibida y el comportamiento real del sujeto en la implantación de determinadas medidas (Coles, Fenclova y Dinan, 2013).

Se sugiere pues que los alojamientos rurales establezcan sistemas de control que evalúen y controlen los beneficios que la asunción de las medidas de RSE suponen para el establecimiento; ello permitirá afianzar unas actitudes más positivas y sólidas hacia las iniciativas sostenibles, no recayendo el peso de su implementación en motivaciones coercitivas o normativas.

En síntesis, el resultado de este trabajo empírico puede considerase satisfactorio al contrastarse positivamente la vinculación entre la IC y la RSE. Ello valida la aplicación y desarrollo de sucesivas investigaciones en el ámbito turístico adoptando la Teoría de la Acción Razonada (TAR) (Fishbein y Ajzen, 1975) y la Teoría del Comportamiento Planeado (TCP) (Ajzen, 1991) como marco teórico para abordar las percepciones de 
los responsables de los alojamientos turísticos hacia la adopción de acciones socialmente responsables desde un punto de vista económico, social y medioambiental.

No obstante, si bien el modelo planteado ha permitido contrastar las hipótesis propuestas, de la interpretación de los resultados se desprenden limitaciones, y en consecuencia propuestas para futuros trabajos. Se señala el carácter no probabilístico del modelo analizado y su influencia en la generalización de los resultados. Futuras investigaciones, en primer lugar, deberían adoptar una metodología cualitativa que complemente la investigación empírica realizada. En segundo lugar, se recomienda analizar el efecto moderador que variables como la dimensión empresarial o el sexo del gerente podrían ejercer en la determinación de posibles diferencias significativas entre distintos grupos en lo que se refiere a las actitudes y el nivel de implementación de acciones socialmente responsables en los alojamientos rurales tal y como sugieren Garay y Font (2013) o Uhlaner et al. (2012), entre otros. Finalmente, es de interés testar el modelo teórico en otros ámbitos geográficos, alojamientos turísticos (hoteles urbanos, litoral) así como extender su aplicación al estudio del punto de vista de la demanda turística.

\section{BIBLIOGRAFÍA}

AJZEN, I. (1991). "The theory of planned behavior". Organization behavior and human decision processes, 50, pp. 179-211.

ALVARADO HERRERA, A. (2008). Responsabilidad Social Percibida desde una perspectiva sostenicéntrica, y su influencia en la reputación de la empresa y en el comportamiento del turista. Tesis Doctoral. Universidad de Valencia, España.

BARCLAY, D., HIGGINS, C. y THOMPSON, R. (1995). “The partial least squares (PLS) approach to causal modeling: Personal computer adoption and use as an illustration". Technology Studies, 2 (2), pp. 285-309.

BENNINGTON, A. J. y MINUTOLO, M. C. (2013). "Intent to be a Socially Responsible Small or Medium-sized Enterprise: Theory of Planned Behavior and Leaders' Actualizing". Journal of Management and Sustainability, 3 (1), pp. 1-8.

CHATZIDAKIS, A., KASTANAKIS, M. y STATHOPOULOU, A. (2016). "Socio-Cognitive Determinants of Consumers' Support for the Fair Trade Movement". Journal of Business Ethics, 133, pp. 95-109.

CHEN, M. F. y TUNG, P. J. (2014). "Developing an extended Theory of Planned Behavior model to predict consumers' intention to visit green hotels". International Journal of Hospitality Management, 36, pp. 221-230.

CHIN, W. W. (1998). "Issues and Opinion on Structural Equation Modeling". MIS Quarterly, 22 (1), pp. 7-15.

CHOU, C. J., CHEN, K. S. y WANG, Y. Y. (2012). "Green practices in the restaurant industry from an innovation adoption perspective: Evidence from Taiwan". International Journal of Hospitality Management, 31, pp. 703-711.

CIAVOLINO, E. y NITTI, M. (2013). "Simulation study for PLS Path Modelling with High- Order Construct: A Job Satisfaction model evidence". Chapter Advanced Dynamic Modeling of Economic and Social Systems. Volume 448 of the series Studies in Computational Intelligence, pp 185-207. 
COLES, T., FENCLOVA, E. y DINAN, C. (2013). "Tourism and corporate social responsibility: A critical review and research agenda". Tourism Management Perspectives, 6, pp. 122-141.

ELKINGTON, J. (1998). Cannibals with forks: The triple bottom line of the 21st century business. Oxford: Capstone Publishing.

FISHBEIN, M. y AJZEN, I. (1975). Beliefs, attitude, intention and behaviour: An introduction to theory and research. Reading Mass: Addison Wesley.

FONT, X., GARAY, L. y JONES, S. (2014). "Sustainability motivations and practices in small tourism enterprises in European protected areas”. Journal of Cleaner Production. Article in Press, pp. 1-10.

FORNELL, C. y LARCKER, D. F. (1981). "Evaluating structural equations models with unobservable variables and measurement error". Journal of Marketing Research, 18, pp. 39-50.

GAO, Y., MATTILA, A. S. y LEE, S. (2016). "A meta-analysis of behavioral intentions for environment-friendly initiatives in hospitality research". International Journal of Hospitality Management, 54, pp. 107-115.

GARAY TAMAJÓN, L. y FONT AULET, X. (2013). “Corporate social responsibility in tourism small and medium enterprises evidence from Europe and Latin America". Tourism Management Perspectives, 7, pp. 38-46.

GRUBER, V. y SCHLEGELMILCH, B. B. (2014). "How Techniques of Neutralization Legitimize Norm- and Attitude-Inconsistent Consumer Behavior”. Journal of Business Ethics, 121, pp. 29-45.

HAIR, J. F., RINGLE, C. M. y SARSTEDT, M. (2011). "PLS-SEM: Indeed a silver bullet". The Journal of Marketing Theory and Practice, 19 (2), pp. 139-152.

HAIR, J. F., SARSTEDT, M., HOPKINS, L. y KUPPELWIESER. V. G. (2014). "Partial least squares structural equation modeling (PLS-SEM): An emerging tool in business research". European Business Review, 26 (2), pp. 106-121.

HAN, H., SHU, L-T. y SHEU, C. (2010). "Application of the Theory of Planned Behavior to green hotel choice: Testing the effect of environmental friendly activities". Tourism Management, 31, pp. 325-334.

HAN, H. y KIM, Y. (2010). “An investigation of green hotel customers' decision formation: Developing an extended model of the theory of planned behavior". International Journal of Hospitality Management, 29, pp. 659-668.

HENSELER, J., RINGLE, C. y SINKOVICS, R. (2009). "The use of Partial of Least Square path modeling in international marketing". Advances in International Marketing, 20, pp. 227-320.

HONG TAN, T. (2013). "Use of Structural Equation Modeling to Predict the Intention to Purchase Green and Sustainable Homes in Malaysia”. Asian Social Science, 9 (10), pp. 181-191.

JASPER, C. R. y WALDHART, P. (2013). "Employer attitudes on hiring employees with disabilities in the leisure and hospitality industry Practical and theoretical implications". International Journal of Contemporary Hospitality Management, 25 (4), pp. 577-594. 
MARTÍNEZ, P., PÉREZ, A. y RODRÍGUEZ DEL BOSQUE, I. (2014). "Exploring the Role of CSR in the Organizational Identity of Hospitality Companies: A Case from the Spanish Tourism Industry". Journal of Business Ethics, 124 (1), pp. 47-66.

MARTÍNEZ GARCÍA DE LEANIZ, P. (2015). Influencia de la responsabilidad social corporativa en el comportamiento del consumidor turístico: un estudio desde la perspectiva del turismo sostenible. Tesis Doctoral. Universidad de Cantabria, España. MONTAÑO VALLE, A. (2015). La estrategia de sostenibilidad como fuente de ventajas competitivas en el sector turístico: modelo de integración de los recursos naturales. Tesis Doctoral. Universidad de Huelva, España.

NUNNALLY, J. C. y BERNSTEIN, I. H. (1994). Psychometric theory (3rd ed.). New York, NY: McGraw-Hill.

OLIVEIRA BROCHADO, F., OLIVEIRA BROCHADO, A. y CALDEIRA, T. (2015). "Os determinantes psicológicos do consumidor verde: the psychological determinants of the green consumer". Tourism and Management Studies, 11 (2), pp. 104-111.

ORGANIZACIÓN MUNDIAL DE TURISMO (O.M.T) (2015). Tourism Highlights, 2015 Edition. Recuperado el 1 de julio de 2016 de: http://goo.gl/b0y2im

ORGANIZACIÓN MUNDIAL DE TURISMO (O.M.T) (2016a). UNWTO World Tourism Barometer. Vol 15. Mayo 2016. Recuperado el 10 de julio de 2016 de: http:// goo.gl/JkAmT

ORGANIZACIÓN MUNDIAL DE TURISMO (O.M.T) (2016b). Sustainable Development of Tourism: mission Statement. Recuperado el 5 de mayo de 2016 de: http:// goo.gl/Rf30Nv

PANWAR, R., RINNE, T., HANSEN, E. y JUSLIN, H. (2006). “Corporate responsibility: Balancing economic, environmental, and social issues in the forest products industry". Forest Products Journal, 56 (2), pp. 4-12.

PEÑA MIRANDA, D. D. y SERRA CANTALLOPS, A. (2012). "Responsabilidad Social Empresarial en el Sector Turístico: estudio de casa en empresa de alojamiento de la ciudad de Santa Marta, Colombia”. Estudios y Perspectivas en Turismo, 21, pp. 1456-1480.

PLAZA ÚBEDA, J. A., DE BURGOS JIMÉNEZ, J., PÉREZ VALLS. M. y ORTEGA EGEA, J. M. (2012). "La percepción directiva de la satisfacción de los grupos de interés". Revista de Responsabilidad Social de la Empresa, 4 (2), pp. 15-44.

PRUD'HOMME, B. y RAYMOND, L. (2013). “Sustainable development practices in the hospitality industry: An empirical study of their impact on customer satisfaction and intentions". International Journal of Hospitality Management, 34, pp. 116-126.

QUINTAL, V. A., LEE, J. A. y SOUTAR, G. N. (2010). "Risk, uncertainty and the theory of planned behavior: A tourism example". Tourism Management, 31, pp. 797-805.

RICHTER, N. F., SINKOVICS, R. R., RINGLE, C. M. y SCHLÄGEL, C. (2016). “A critical look at the use of SEM in international business research". International Marketing Review, 33 (3), pp. 376-404.

ROLDÁN, J. L. y SÁNCHEZ FRANCO, M. J. (2012). "Variance-Based Structural Equation Modeling: Guidelines for Using Partial Least Squares in Information 
Systems Research". In Mora, M., Gelman, O., Steenkamp, A. y Raisinghani, M.S (eds). Research Methodologies, Innovations and Philosophies in Software Systems Engineering and Information Systems, pp. 193-221.

SANAGUSTÍN FONS, M. V., MOSEÑE FIERO, J. A. y GOMEZ Y PANIÑO, M. (2011). "Rural tourism: a sustainable alternative". Apply Energy, 88, pp. 551-557.

SÁNCHEZ FERNÁNDEZ, M. D. (2014). "Institutional context of hotel social responsibility in the Euro-region: a factorial analisis". Revista de Ocio y Turismo (ROTUR), 7, pp. 106-119.

SARSTEDT, M., RINGLE, C. M. y HAIR, J. F. (2014). "PLS-SEM: Looking Back and Moving Forward". Long Range Planning, 47 (3), pp. 132-137.

SARSTEDT, M., HAIR, J. F., RINGLE, C. M., THIELE, K. O. y GUDERGAN, S. P. (2016). "Estimation issues with PLS and CBSEM: Where the bias lies!". Journal of Business Research, 69, pp. 3998-4010.

SHARMA, P. y SHARMA, S. (2011). "Drivers of proactive environmental strategy in family firms". Business Ethics Quarterly, 21 (2), pp. 309-334.

STAHL, G. K. y SULLY DE LUQUE, M. (2014). "Antecedents of responsible leader behavior: a research synthesis, conceptual framework, and agenda for future research". The Academy of Management Perspectives, 28 (3), pp. 235-254.

TENG, Y. M., WU, K. S. y LIU, H. H. (2015). "Integrating altruism and the Theory of Planned Behavior to predict patronage intention of a green hotel". Journal of Hospitality and Tourism Research, 39 (3), pp. 299-315.

TILIKIDOU, I., DELISTAVROU, A. y SAPOUNTZIS, N. (2014). “Customers' Ethical Behaviour towards Hotels". Procedia Economics and Finance, 9, 425-432.

UHLANER, L. M., BERENT-BRAUN, M. M., JEURISSEN, R. J. M. y DE WIT, G. (2012). "Beyond Size: Predicting Engagement in Environmental Management Practices of Dutch SMEs". Journal Business Ethics, 109, pp. 411-429.

VACA ACOSTA, R. M. (2013). Responsabilidad social corporativa en las cadenas hoteleras españolas: factores determinantes de su nivel de desarrollo y consecuencias. Tesis Doctoral. Universidad de Huelva, España.

VAN MARREWIJK, M. (2003). "Concepts and definitions of CSR and corporate sustainability: Between agency and communion". Journal of Business Ethics, 44 (2), pp. 95-105.

VARGAS SÁNCHEZ, A., VACA ACOSTA, M. A. y GARCÍA DE SOTO CAMACHO. E. (2004). Turismo rural y medio ambiente. Diagnostico medioambiental de los alojamientos rurales de la provincia de Huelva. Fundación Biodiversidad.

WILLIAMS, L., VANDENBERG, R. J. y EDWARDS, R. J. (2009). "Structural Equation Modeling in Management Research: A guide for improved analysis". The Academy of Management Annals, 3 (1), pp. 543-604.

WOJTAROWSKI LEAL, A., SILVA RIVERA, E., PIÑAR ALVAREZ, M. A. y NEGRETE RAMÍREZ, J. A. (2016). "La Responsabilidad Social Empresarial como pieza clave en la transición hacia el desarrollo sustentable en el sector turístico". Revista Pasos, 14 (1), pp. 127-139. 
WORLD BUSINESS COUNCIL ON SUSTAINABLE DEVELOPMENT (WBCSD) (2016). Corporate social responsibility: Meeting changing expectations. Recuperado el 3 de julio de 2016 de: http://goo.gl/uNr7Jx

WRIGHT, R. T., CAMPBELL, D. E., THATCHER, J. B. y NICHOLAS, R. (2012). "Operationalizing Multidimensional Constructs in Structural Equation Modeling: Recommendations for IS Research". Communications of the Association for Information Systems, 30 (23), pp. 367-412.

YACOB, P. y MOORTHY, M. K. (2012). "Green Practices: Perception of Malaysian SME Owners/Managers". International Journal of Academic Research in Economics and Management Sciences, 1 (3), pp. 103-111.

YACOB, P., BINTI AZIZ, N. S., MOHAMAD MAKMOR, M. F. y MOHD ZIN. A. W. (2013). "The policies and green practices of Malaysian SMEs". Global Business and Economics Research Journal, 2 (2), pp. 52-74.

ZIENTARA, P., KUJAWSKI, L. y BOHDANOWICZ, P. (2015). "Corporate social responsibility and employee attitudes: evidence from a study of Polish hotel employees". Journal of Sustainable Tourism, 23 (6), pp. 859-880. 